\title{
EVIDENCIAS DE VALIDEZ Y CONFIABILIDAD DE LA ESCALA DE FLEXIBILIDAD EN JÓVENES MEXICANOS
}

\author{
JORGE PALACIOS \\ http://orcid.org/0000-0002-4351-5667 \\ Universidad del Valle de México, Querétaro, México \\ Correo electrónico: jorge.palaciosd@uvmnet.edu
}

Recibido: 18 de enero del 2021 / Aceptado: 1 de marzo del 2021

doi: https://doi.org/10.26439/persona2021.n024(1).5311

\begin{abstract}
Resumen. El objetivo de la investigación fue analizar la confiabilidad y validez de las puntuaciones de la escala de flexibilidad de Melgoza y Díaz-Guerrero (1990) en jóvenes mexicanos. Se aplicó la escala a una muestra de 309 jóvenes, de entre 14 y 24 años de edad, de la zona metropolitana de la Ciudad de México. Los resultados muestran la estructura factorial integrada por tres factores mediante un análisis factorial confirmatorio, referentes a la amabilidad, complacencia y adaptación conductual. Particularmente la amabilidad $(\beta=.325, p<.001)$ y la adaptación conductual $(\beta=.245, p<.001)$ son predictores de la eficiencia personal. Las puntuaciones de la escala alcanzan un índice de consistencia interna de .85 (IC95 \% = .83 - .87). Se analiza la utilidad de los resultados para la evaluación de la flexibilidad y los rasgos de personalidad en los jóvenes, así como las implicaciones para futuras investigaciones sobre etnopsicología.
\end{abstract}

Palabras clave: escala de flexibilidad / rasgo de personalidad / etnopsicología y jóvenes

\section{EVIDENCE OF VALIDITY AND RELIABILITY OF THE FLEXIBILITY SCALE IN MEXICAN YOUTH}

Abstract. The purpose of this study was to analyze validity and reliability of the scores obtained with the Scale of the Flexibility (Melgoza y Díaz-Guerrero, 1990) in a sample of Mexican youth. The scale was tested in a sample of 309 young people between 14 and 24 years old, in México City and Metropolitan Area. Confirmatory factor analysis showed a three-factor structure: kindness, complacency and behavioral adaptation. Particularly kindness $(\beta=.325, p<.001)$ and behavioral adaptation $(\beta=.245, p<.001)$ predicted personal efficiency. The scale score has an internal consistency index of $.85(95 \% \mathrm{Cl}=.83-.87)$. The usefulness of the results for the evaluation of flexibility and personality traits in young people is analyzed, as well as the implications for future research on ethnopsychology.

Keywords: flexibility scale / personality trait / ethnopsychology and youths 


\section{INTRODUCCIÓN}

Existen dos aproximaciones empleadas para comprender la estructura de la personalidad a través de diferentes culturas (Church, 2000, 2001; McCrae, 2001). La primera es la aproximación Etic que usualmente emplea mediciones transculturales centradas en constructos que pretenden contener rasgos universales. Diferentes investigaciones realizadas en México bajo el enfoque Etic han encontrado que algunos rasgos universales como el locus de control (La Rosa, Díaz-Loving y Andrade, 1986), la asertividad (Flores, Díaz-Loving y Rivera, 1987), la búsqueda de sensaciones (Palacios, 2015) o el autoconcepto (La Rosa y Díaz-Loving, 1991; Valdez y Reyes, 1993) presentan algunas variaciones etnoculturales que no se ajustan adecuadamente en nuestro país, lo que ha llevado a proponer que al momento de retomar rasgos universales se debe considerar la sensibilidad cultural en su medición.

La segunda aproximación del estudio de la personalidad es el Emic, que investiga rasgos idiosincráticos (indígenas) particulares de cada cultura (Díaz-Guerrero, 1994; Díaz-Loving, 1998; Reyes, 1996). En México, la investigación realizada bajo esta aproximación denominada "enfoque etnopsicológico" ha encontrado rasgos autóctonos (indígenas) que parecen ser cardinales de la personalidad del mexicano. Entre estos rasgos destacan la abnegación (Avendaño y Díaz-Guerrero, 1992), la flexibilidad (Melgoza y Díaz-Guerrero, 1990), los tipos de mexicano (Díaz-Guerrero, 1994) y dimensiones emergentes de la personalidad del mexicano (Palacios y Martínez, 2017). Estos estudios han permitido identificar constructos etnopsicológicos de la personalidad del mexicano, que puede observarse en el estudio sistemático del autoconcepto (Cortés y Flores, 2002; Díaz-Loving, Reyes-Lagunes y Rivera-Aragón, 2002; La Rosa y Díaz-Loving, 1991; Valdez, González, Jiménez y Cañas, 1996; Valdez y Reyes, 1992; Valdez y Reyes, 1993). En estos estudios se han logrado observar las características del mexicano y su relación con rasgos propios de esta cultura.

Para Díaz-Guerrero (1996), la personalidad del mexicano emerge al asumir los mandatos de su cultura por medio del grado de aceptación o rechazo de premisas socioculturales (PSC), que inciden en el comportamiento individual (Díaz-Guerrero, 1988, 2008). En términos de sus características individuales, el mexicano aprende que existen dos maneras de encarar los problemas de la vida (estilo de confrontación). Uno es de forma activa, es decir, modificando el ambiente; el otro, de forma pasiva, adaptándose a los problemas, modificando el propio yo, más que al medio ambiente a fin de enfrentar las dificultades (Díaz-Guerrero, 1967, 1984, 1994). La forma pasiva de confrontación parece ser un factor cardinal en la personalidad de los mexicanos (Díaz-Guerrero, 1988, 1993) comparado con culturas individualistas (Díaz-Guerrero, 1994; Triandis, 1996).

Los estudios realizados sobre las dimensiones fundamentales de la personalidad del mexicano han encontrado que para funcionar conductualmente en la cultura mexicana, 
los individuos tienden a comportarse de manera obediente, afiliativa y respetuosa de las normas (Díaz-Guerrero, 1984, 1993, 1996), así como a desarrollar idiosincrásicamente rasgos socioexpresivos y expresivo-normativos (La Rosa y Díaz-Loving, 1991; DíazLoving, 1998) que describen a los mexicanos como sociables, amables y fáciles de tratar (Valdez y Reyes, 1993), lo que les permite ser aceptados y obtener ciertas oportunidades (Díaz-Guerrero, 1988, 1994, 1995, 2001).

A partir de las características descritas por los mexicanos, uno de los rasgos de personalidad que parece ser relevante para la cultura mexicana es la flexibilidad. Según la Real Academia de la Lengua Española (2015), la flexibilidad involucra: 1) la disposición para doblarse fácilmente; 2) plegarse con facilidad ante un enfrentamiento, a la opinión, voluntad o a la actitud de otro o de otros (persona de carácter flexible); y 3) es susceptible de cambios o variaciones según las circunstancias o necesidades. Para Coppens, de Boer y Koolhaas (2010), la flexibilidad conductual es definida como la habilidad de un individuo para responder directamente y ajustar su conducta frente a un estímulo medioambiental.

Según la psicología, la flexibilidad puede ser medida y abordada desde diversas perspectivas, como son el afrontamiento (Cheng y Cheung, 2005), las estrategias de adaptación (Buss, 1991, 2009; Buss y Greiling, 1999; Palacios, Bustos y Mercado, 2013; Palacios, Bustos y Soler, 2015), la flexibilidad cognitiva (Albanesi, Garelli y Masramon, 2009; Coppens et al., 2010), la adaptación (Buss, 1996, Buss y Grelling, 1999; Palacios et al., 2013), la neurocognición (Roberts, Barthel, López, Tchanturia y Treasure, 2011; Palacios, Ramírez, Hernández y Anaya, 2016) o como característica de personalidad (Bond, Lloyd y Guenole, 2013; Williams, Ciarrochi y Heaven, 2012).

Melgoza y Díaz-Guerrero (1990) abordaron la flexibilidad del mexicano y desarrollaron una escala de flexibilidad, considerándola como un rasgo cardinal en la personalidad del mexicano. Realizaron un análisis factorial en una muestra de 80 maestros de la Ciudad de México, obteniendo tres factores claramente interpretables: 1) amabilidad, 2) condescendencia y 3) flexibilidad. La confiabilidad a través del coeficiente alfa de Cronbach para las tres dimensiones fue de .75, .56 y .74, respectivamente. En los 80 casos estudiados, la puntuación promedio más alta fue la amabilidad, seguida de la condescendencia y la flexibilidad. Los hombres son más amables y condescendientes que las mujeres y estas más flexibles que los varones. Se ha hipotetizado que las dimensiones de la flexibilidad se correlacionan con rasgos propios de la cultura mexicana (Díaz-Guerrero, 1994; Melgoza y Díaz-Guerrero, 1990) como son la obediencia afiliativa, la no asertividad y la abnegación; se espera que en rasgos como la severidad, la eficiencia y el individualismo se obtengan relaciones negativas, sin embargo, estos supuestos no se han investigado.

Varela-Macedo, Díaz-Loving y Reyes-Lagunes (1998) utilizaron el instrumento de Melgoza y Díaz-Guerrero (1990) para analizar el proceso de aculturación de estudiantes 
extranjeros que migran a México y encontraron que la flexibilidad se relaciona con la asimilación, integración y dominio del idioma. Esto indica las características para afrontar el proceso de aculturación, se requiere de flexibilidad para adaptar los elementos propios de su cultura de origen y aquellos pertenecientes a la cultura huésped. Por su parte, Ortiz, Church, Vargas, Ibáñez, Flores luit y Escamilla (2007) tuvieron como objetivo replicar varias dimensiones autóctonas (indígenas) de la personalidad, entre ellas la flexibilidad. Los autores estimaron la confiabilidad y realizaron un análisis factorial de la escala en 794 mexicanos de las zonas centro y sur de México. Primero compararon el coeficiente de confiabilidad alfa de Cronbach obtenido en su muestra total de mexicanos con lo reportado por el autor original. Los niveles de confiabilidad alfa de Cronbach obtenidos en su muestra para las tres dimensiones de la flexibilidad fueron de .57 , .43 y .61, respectivamente. Al no obtener valores óptimos de confiabilidad, realizaron un análisis factorial de ejes principales para obtener la representación más replicable del instrumento. Los autores encontraron cargas duales en su estructura, fallando en replicar las tres dimensiones originales, por lo que optaron por una solución de un solo factor. La confiabilidad por consistencia interna (alfa de Cronbach) para el puntaje total de la escala fue de .74 .

La evidencia mostrada hasta el momento señala que la personalidad emerge en interacción con la sociocultura en la que se encuentra el individuo, particularmente la personalidad del mexicano incluye rasgos socioexpresivos, obedientes-afiliativos y con una tendencia para ajustarse a su entorno. Adicionalmente, se destaca que existen pocos estudios que empíricamente evalúen el rasgo de flexibilidad en nuestro país. Lo encontrado muestra resultados inconsistentes que permitan entender la cercanía del individuo con la cultura tradicional mexicana o qué tanto se ha alejado de ella, por medio de rasgos de personalidad que faciliten indagar su incidencia en las relaciones interpersonales, sus normas, la forma de comportarse y en conductas relacionadas con su bienestar. Además, parece oportuno someter a prueba la estabilidad psicométrica de la escala de flexibilidad elaborada por Melgoza y Díaz-Guerrero (1990), utilizando procedimientos adicionales que le otorguen nuevas evidencias de validez en nuestro país y en una muestra de jóvenes a diferencia de la realizada en su versión original que fue en profesores de bachillerato. Considerando que el rasgo de flexibilidad tiene un correlato sociocultural y que en México los jóvenes son el mayor grupo poblacional, es relevante evaluar las facetas de la flexibilidad que pueden manifestar los adolescentes y las conductas posteriores que se pueden presentar en el transcurso de su desarrollo. Con la finalidad de dar continuidad a lo encontrado en México (Melgoza y Díaz-Guerrero, 1990; Ortiz et al., 2007), se decidió realizar la presente investigación con el objetivo de obtener evidencias de confiabilidad y validez (relacionadas con la estructura interna y a partir de las relaciones con otras variables) para el uso de la escala de flexibilidad en una muestra de jóvenes mexicanos. 
Partiendo de la noción de que la cultura mexicana forma parte de los países colectivistas, tiende a utilizar y priorizar normas del grupo para moldear la conducta y mantener sus relaciones sociales, muestra cooperación entre sus miembros (DíazGuerrero, 1994), valora menos acciones individuales, percibe menos control personal del medio y posee una imagen de sí más dependiente del grupo (Singelis, Triandis, Bhawuk y Gelfand, 1995; Triandis, 1994, 2000, 2001); se considera que la flexibilidad puede ser un rasgo positivo para el individuo o la sociocultura mexicana, ya que esta promueve características expesivo-afiliativas y socioafectivas en los mexicanos, en contraparte con características como la competitividad, la eficiencia o el logro de metas, propias de culturas individualistas. Por lo tanto, se plantea que la flexibilidad que los jóvenes poseen influirá en la capacidad de eficiencia personal (Palacios, 2014) para realizar o cumplir adecuadamente una función y obtener un resultado, como se ha indicado en estudios antecedentes (Díaz-Guerrero, 1994), lo que otorgará evidencias predictivas de validez.

\section{MÉTODO}

\section{Diseño}

Para la presente investigación se realizó un estudio de campo con un diseño de investigación no experimental de corte transversal, instrumental y psicométrico (Montero y León, 2002).

\section{Participantes}

Se seleccionó una muestra no probabilística de tipo intencional de 309 jóvenes, 112 hombres y 197 mujeres, con un rango de edad entre 14 y 24 años y una media de 16,70 años ( $D E=1,7)$, estudiantes de dos escuelas públicas de educación media superior de la zona metropolitana de la Ciudad de México. Se consideraron como criterios de inclusión estar inscrito en bachillerato, desear participar en la investigación y no presentar algún problema cognitivo.

\section{Instrumentos}

1. Escala de flexibilidad (Melgoza y Díaz-Guerrero, 1990). A la versión original de los autores se le realizaron algunas adecuaciones, modificando la redacción de las afirmaciones para ajustarlas al vocabulario de los jóvenes. Por ejemplo, el ítem "Soy acomodaticio" se cambió por el ítem "Me acomodo a las situaciones", al ítem "Me gusta complacer" se le agregó la frase "a las personas", se eliminaron los ítems "Soy condescendiente", “Soy servicial" y se incluyó el ítem "Soy flexible". Los ítems modificados se redactaron haciendo énfasis en que su contenido fuera congruente con la etnopsicología de la sociocultura mexicana (Díaz-Guerrero, 1977, 1984, 1994, 1996; Díaz-Loving, 1998; 
Palacios y Martínez, 2017) utilizada en el presente estudio. Adicionalmente, se cambió el formato de respuesta tipo diferencial semántico, por uno tipo Likert. El instrumento final estuvo conformado por catorce afirmaciones con cuatro opciones de respuesta cerrada tipo Likert que va de "casi nunca" a "siempre". Una mayor puntuación representa ser más flexible y adaptada conductualmente a la sociocultura.

Para este estudio el rasgo de flexibilidad implica que los jóvenes modifiquen, cambien o se adapten con facilidad a las personas o a la interacción con los otros, utilizando características socioexpresivas de amabilidad, agrado y colaboración, de acuerdo a las circunstancias, necesidades del contexto o a las demandas de la situación.

2. Escala de eficiencia personal (Palacios, 2014). Es una escala unifactorial tipo Likert de seis ítems, en la que los participantes reportan la capacidad para ser eficiente, organizado y perseverante en las actividades que realiza cotidianamente (por ejemplo, "Me considero responsable"). Se contesta con cuatro opciones de respuesta que van de "nunca" hasta "siempre". El autor ha reportado un coeficiente de confiabilidad de .81 (IC95\% = .77 - .84) para el total del instrumento, así como evidencias de validez basadas en el contenido y a partir de las relaciones con variables como el locus de control financiero (Palacios y Soler, 2017).

\section{PROCEDIMIENTO}

En primer lugar, se pidió autorización a las instituciones para realizar la investigación. La aplicación de la escala y la obtención de información se logró a lo largo de un mes que duró la investigación. Se solicitó la firma de asentimiento informado y se aplicó el instrumento realizado en lápiz y papel a los adolescentes de manera grupal, utilizando a los grupos escolares para tal fin. Asimismo, se les pidió que respondieran a un cuestionario elaborado para conocer algunas actividades que realizan los jóvenes de su edad.

\section{Consideraciones éticas}

A todos los participantes se les aclaró que su participación era voluntaria y que la información era anónima, se les garantizó la confidencialidad de los datos proporcionados y se resolvieron las dudas que tuvieran. Se utilizó el consentimiento informado de los participantes y de las autoridades escolares. El protocolo de investigación fue establecido de acuerdo al Reglamento de la Ley General de Salud, en su apartado sobre investigación en seres humanos (Secretaría de Salud, 2011).

\section{RESULTADOS}

Para evaluar las características psicométricas del instrumento, se empleó la siguiente estrategia de análisis de datos: en primer lugar, se revisó la distribución de frecuencias 
de las respuestas de cada ítem dadas por los jóvenes para conocer si dichos ítems discriminaban, considerando que tuvieran la distribución de las respuestas cercanas a una curva normal, eliminando los ítems que tuvieran más del $80 \%$ en una sola opción de respuesta, indicativo de que la mayoría de los sujetos respondían en los extremos de la escala de respuesta y, por tanto, resultaban sesgados; de este modo se buscaba eliminar aquellos ítems que tuvieran una distribución asimétrica. A continuación, se realizó un análisis de discriminación de reactivos a través de la prueba $t$ de Student; en este caso, se tomó en cuenta que la probabilidad fuera de .05 o menor. El siguiente criterio para la eliminación de reactivos fue la correlación ítem-total, eliminando aquellos que obtuvieran una correlación menor a .20. La discriminación de reactivos permitió mantener a los 14 ítems de la escala original.

Para obtener evidencias de validez basadas en la estructura interna de la escala de flexibilidad y someter a prueba la estructura de tres factores obtenida por Melgoza y Díaz-Guerrero (1990) (ver tabla 1), se realizó un análisis factorial confirmatorio (figura 1) con el programa AMOS (Arbuckle y Wothke, 1999). Los análisis realizados en la muestra de estudio mostraron un ajuste óptimo a los datos, proporcionando validez a la medida. Los indicadores de bondad de ajuste absoluto $\left[X^{2}(73, N=309)\right.$ $=182.44, p=.00 ;$ RMSEA $=.07$ ]; de ajuste incremental $(T L I=.88 ; \mathrm{RFI}=.82 ; \mathrm{IFI}=.91$; $\mathrm{CFI}=.90)$ y de ajuste de parsimonia (NFI = .86) mostraron estar cercanos a lo aceptable (Hair, Anderson, Tatham y Black, 1999; Martínez, Hernández y Hernández, 2006; Pérez, Chacón y Moreno, 2000). Asimismo, los pasos factoriales de cada indicador en el factor fueron elevados y estadísticamente significativos $(p<.05)$

Tabla 1

Ítems de la escala de flexibilidad

Amabilidad
25. Me gusta ser amable
29. Me gusta ser generoso(a)
26. Me gusta cooperar con los demás
28. Me gusta servir a los demás
24. Soy tolerante
Condescendencia
23. Me gusta complacer a las personas
34. Me gusta consentir a las personas
30. Me gusta agradarle a los demás
33. Soy blando(a) en mi forma de ser
32. Me agrada conceder (darle su lugar a los otros/as)

(continúa) 
(continuación)

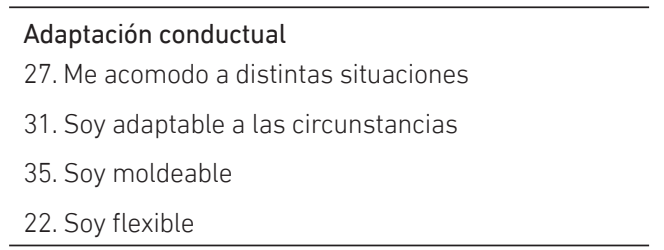

Elaboración propia

Los factores de la escala de flexibilidad quedaron definidos de la siguiente forma. El primer factor se denominó "Amabilidad", está integrado por cinco aseveraciones que implican acciones de buen trato y cooperación con las demás personas. La segunda dimensión, nombrada "Condescendencia", agrupó cinco afirmaciones e implica el gusto de los jóvenes por complacer o dar gusto a las personas, por tratar de agradar al otro, involucrando acciones para dar gusto a los demás. Por último, la faceta de adaptación conductual se refiere a las características que un individuo posee para acomodarse o ajustarse a diversas circunstancias o condiciones de su entorno, y se encuentra integrada por cuatro aseveraciones.

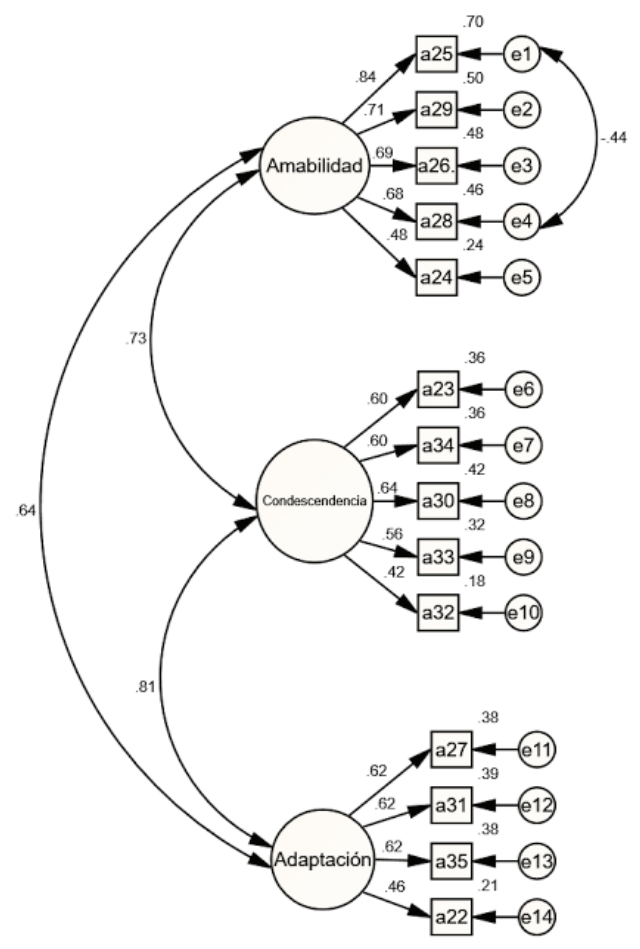

Figura 1. Análisis factorial confirmatorio de la escala de flexibilidad Elaboración propia 
Adicionalmente, se calculó la confiabilidad de las puntuaciones de la escala con dos métodos, división por mitades y consistencia interna (alfa de Cronbach) para el total del instrumento y para cada faceta obtenida. El coeficiente de correlación obtenido entre las dos mitades fue de .699 , con la fórmula de corrección de Spearman-Brown de igual longitud fue de .823 y mediante la fórmula de Guttman se obtuvo un valor de .823. El análisis de consistencia interna para el puntaje total mostró un coeficiente alfa de Cronbach de .85 (IC95 \% = .83 - .87). El intervalo de confianza de la fiabilidad para el instrumento en su totalidad no disminuye de .70 y no supera el .90 , indicando la estabilidad de la escala.

El análisis descriptivo (media aritmética, desviación estándar, rango, número de ítems y consistencia interna) obtenido de cada faceta de la escala se encuentra en la tabla 2. Los valores promedio están por encima de la media teórica, lo cual indica los niveles de flexibilidad en las respuestas de los jóvenes. Las desviaciones estándar son constantes a través de los factores. La puntuación promedio más alta fue la amabilidad, seguida de la condescendencia y, por último, la adaptación conductual.

Tabla 2

Estadísticos descriptivos de la escala de flexibilidad

\begin{tabular}{lccllrl}
\hline & $\mathrm{M}$ & $\mathrm{DE}$ & Ítems & Rango & $\alpha$ & IC95\% \\
\hline Amabilidad & 2,93 & 0,6 & 5 & $1-4$ & .78 & $.74-.82$ \\
Condescendencia & 2,74 & 0,6 & 5 & $1-4$ & .69 & $.64-.74$ \\
Adaptación conductual & 2,67 & 0,6 & 4 & $1-4$ & .66 & $.59-.72$ \\
\hline
\end{tabular}

Elaboración propia

Para comparar la puntuación media de cada faceta entre hombres y mujeres, se utilizó la prueba $t$ de Student. Los resultados mostraron diferencias estadísticamente significativas entre hombres y mujeres solo en el factor de amabilidad (hombres: $M=2,78, D E=0,7$; mujeres: $\left.M=3,01, D E=0,6 ; t=2,84, p<.01, \eta^{2}=0,33\right)$, en donde las mujeres tienen un puntaje más alto en amabilidad en comparación con los hombres. La condescendencia (hombres: $M=2,69, D E=0,6$; mujeres: $M=2,77, D E=0,6 ; t=1,31$, $p=0,25, \eta^{2}=0,04$ ) y la adaptación conductual (hombres: $M=2,65, D E=0,6$; mujeres: $\left.M=2,67, D E=0,6 ; t=0,26, p=0,78, \eta^{2}=0,00\right)$ no fueron diferentes estadísticamente.

En seguida, se calculó el coeficiente de correlación $r$ de Pearson entre la puntuación de las tres facetas obtenidas de la flexibilidad. La tabla 3 muestra que los factores se correlacionan de forma positiva y estadísticamente significativa. La amabilidad correlaciona significativamente con la condescendencia y con la adaptación conductual, en la misma dirección y en menor magnitud con la adaptación conductual. Esta última faceta 
de la flexibilidad se relacionó positiva, significativamente y con similar magnitud con la amabilidad y la condescendencia. Los niveles de correlación permiten asumir que son dimensiones cercanas entre sí y posibles rasgos cardinales en nuestro país.

Tabla 3

Correlaciones entre los factores de la escala de flexibilidad

\begin{tabular}{lccc}
\hline & \multicolumn{3}{c}{ Adaptación } \\
\cline { 2 - 4 } & Amabilidad & Condescendencia & Conductual \\
\hline Amabilidad & -- & $.568^{*}$ & $.508^{*}$ \\
Condescendencia & & -- & $.568^{*}$ \\
Adaptación conductual & & & -- \\
\hline
\end{tabular}

${ }^{*} p<.001$

Elaboración propia

Una vez establecida la dimensionalidad de la escala de flexibilidad, así como las relaciones entre los factores, se consideró obtener evidencias de validez referida a un criterio (predictiva) mediante un análisis de regresión múltiple de pasos sucesivos, calculando el peso de cada una de las tres facetas de la flexibilidad en la puntuación total de la eficiencia. En el análisis de regresión se utilizaron como predictores las tres dimensiones de la flexibilidad y se consideró como criterio el nivel de eficiencia que tuvieran los jóvenes.

Tabla 4

Análisis de regresión múltiple paso por paso para predecir la eficiencia personal

\begin{tabular}{lccc}
\hline Variable & B & SE B & $\beta$ \\
\hline Paso 1 & & & \\
$\quad$ Amabilidad & 1.80 & 0.32 & $.325^{*}$ \\
Paso 2 & & & \\
$\quad$ Adaptación conductual & 1.42 & 0.33 & $.245^{*}$ \\
\hline
\end{tabular}

${ }^{*} p<.001$

Elaboración propia

Los resultados obtenidos mostraron que para la eficiencia se identificaron dos predictores independientes y significativos que entraron en el modelo de regresión. En el primer paso entró la amabilidad $(F=77,51, p<.001)$ como el mejor predictor; en el segundo paso, se incorporó la adaptación conductual en la ecuación de regresión ( $F=$ $49,94, p<.001)$. Lo anterior significa que los jóvenes que son amables con los demás individuos y que adaptan su comportamiento al contexto sociocultural realizan más 
actividades para ser considerados como eficientes. Ambas características del rasgo de flexibilidad explican el $24,6 \%$ de la varianza de la eficiencia de las personas (ver tabla 4).

\section{DISCUSIÓN}

Se evaluaron diferentes aspectos de la validez para el uso de la escala de flexibilidad (Martínez et al., 2006; Pérez et al., 2000). Los resultados obtenidos proporcionan apoyo a la validez de la medida de flexibilidad a través de tres facetas (amabilidad, condescendencia y adaptación conductual), respaldando la estructura factorial obtenida por Melgoza y Díaz-Guerrero (1990), no así con la noción que señala que la flexibilidad puede ser integrada en una sola dimensión como lo señalan Ortiz et al. (2007). Los pesos factoriales elevados y relaciones significativas entre cada factor (faceta) y los indicadores que la componen reafirman que la flexibilidad puede ser medida por una serie de ítems contenidos por tres facetas que le otorgan congruencia conceptual, como se evaluaron en el presente estudio. Los resultados de los diferentes niveles de ajuste del análisis factorial confirmatorio se encuentran cercanos a lo aceptable, lo que permite interpretar que el modelo factorial sugerido es válido; sin embargo, existe la posibilidad de hacer adecuaciones a los ítems que le permitan obtener un mejor ajuste a los datos.

En términos teóricos, el rasgo de flexibilidad describe un aspecto instrumental caracterizado por la faceta de adaptación conductual; el segundo aspecto que describe este rasgo es la expresividad que se encuentra representada por la faceta de condescendencia y principalmente en la faceta de amabilidad (Melgoza y Díaz-Guerrero, 1990). El hecho de corroborar facetas de personalidad como las aquí descritas evidencia la presencia de rasgos del mexicano (Díaz-Guerrero, 1994; Palacios, 2014; Palacios y Martínez, 2017) bajo el enfoque etnopsicológico. Los rasgos de personalidad identificados en el presente estudio pueden ser entendidos como rasgos relativamente permanentes de la personalidad de un individuo, que se manifiestan en diferentes situaciones y que se representan por medio de facetas que llevan a cabo las personas (Palacios, 2015).

A partir de los datos encontrados, la faceta de amabilidad explica la mayor variabilidad de la flexibilidad; estas características que los jóvenes señalan poseer encuentran un amplio respaldo bajo el enfoque etnopsicológico. Esta faceta es congruente con lo encontrado en otros estudios sobre los tipos de mexicano (Palacios, 2014), con el estudio sistemático del autoconcepto del mexicano (Cortés y Flores, 2002; Díaz-Loving et al., 2002; La Rosa y Díaz-Loving, 1991; Valdez et al., 1996; Valdez y Reyes, 1992, 1993), así como con la descripción de las características de personalidad de los mexicanos (Díaz-Guerrero, 1994; Palacios y Martínez, 2017) al referir que para los mexicanos es fundamental ser expresivos y que es necesario ser agradables, debido a que tiene un alto beneficio social.

La faceta de condescendencia es una de las características que está fundamentalmente vinculada a la sociocultura mexicana, es decir, que los jóvenes manifiestan 
complacer, consentir y agradar a las personas, representando la manera en que se comporta el mexicano dentro de su familia y que le sirve para convivir en la sociedad en la que crece y se desarrolla (Palacios et al., 2015). Los hallazgos de esta categoría son congruentes con estudios (Avendaño y Díaz-Guerrero, 1992; Díaz-Guerrero, 1993, 1994, 1996) que mencionan que la abnegación es una característica de las más comunes en la sociedad mexicana y que refleja la forma apropiada de relacionarse con el grupo de referencia (familia) del mexicano. Además, el contenido de esta faceta se encuentra relacionado con el tipo de mexicano obediente afiliativo (Díaz-Guerrero, 1994; Palacios y Martínez, 2017), así como el lado contrario de la característica de personalidad rebelde de Palacios y Martínez (2017).

Por último, la faceta de adaptación conductual retoma las características de ajuste que los jóvenes utilizan ante exigencias que provienen del medio social. La adaptación, como se habrá observado, es personal y se refuerza culturalmente en donde los jóvenes se adaptan a las modificaciones ambientales existentes (Palacios et al., 2013). La adaptación conductual incluye algunos descriptores del lado pasivo de la dicotomía activo-pasiva (Díaz-Guerrero, 1967, 1977, 1984, 1994), es decir, se observa con claridad que la adaptación conductual de la flexibilidad constituye la forma pasiva para adaptarse a los problemas, modificando el propio yo, a fin de enfrentar las dificultades que se presentan (Díaz-Guerrero, 1984, 1993). Las características de esta faceta de la flexibilidad encuentran apoyo en otros autores (Buss, 1991, 2009: Buss y Greiling, 1999; Coppens et al., 2010; Palacios et al., 2013; Palacios et al., 2015) al mencionar que la adaptación a un contexto requiere un cambio en el comportamiento para poder habituarse al nuevo ambiente.

Para obtener evidencias predictivas de validez de la medición de la flexibilidad en esta publicación, se utilizó como criterio la eficiencia personal, tal como lo proponen otros estudios (Díaz-Guerrero, 1994; Palacios y Soler, 2017). Los datos encontrados sugieren que las facetas de amabilidad y adaptación conductual son predictores de la eficiencia personal; es decir, los jóvenes que por un lado son amables, generosos o cooperan con los demás, y que por otra parte, son moldeables, se acomodan a los problemas cuando se les presentan, ajustando su forma de ser de acuerdo a la situación, son los que realizan más acciones para ser eficientes, aumentando su capacidad para realizar o cumplir adecuadamente una función y obtener un resultado en su vida; este efecto apoya lo encontrado por otros estudios (Díaz-Guerrero, 1994; La Rosa et al., 1986; Palacios, 2014; Palacios y Soler, 2017) al mencionar que a una persona con algunas o con todas estas características se le abren las oportunidades para obtener o lograr resultados óptimos en su vida.

Los hallazgos obtenidos en el modelo de regresión permiten sustentar que la flexibilidad tiene un efecto en la capacidad de eficiencia personal de los jóvenes; de esta forma, con los datos mostrados, es posible sostener que la flexibilidad es un predictor de 
la eficiencia personal en los jóvenes de esta muestra. En este sentido, los jóvenes pueden utilizar la amabilidad y la adaptación conductual para alcanzar metas o lograr obtener algún tipo de éxito. La obtención de logros vinculados con ser eficiente depende de la forma en la que los jóvenes ajustan o moldean su comportamiento en las situaciones en las que se encuentren; además, los jóvenes consiguen sus objetivos a través de relaciones afectivas con quienes les rodean. Los resultados obtenidos encuentran respaldo con estudios previos (Díaz-Guerrero, 1994, 1995, 1996; Díaz-Loving, 1998; La Rosa et al., 1986; Palacios y Soler, 2017) que señalan que el mexicano integra las cualidades de su cultura, al ser afectuoso y cortés con los demás, adicionando un estilo de automodificación para adaptarse a su entorno (Díaz-Guerrero, 1994) y para obtener algún logro utiliza las relaciones con las otras personas para conseguirlo.

Con lo encontrado en el presente estudio sobre el rasgo de flexibilidad y su efecto en la eficiencia, se aporta evidencia a la etnopsicología (Díaz-Guerrero, 1977, 1984, 1988, 1994, 2001, 2008; Díaz-Loving, 1998) y a los rasgos centrales de la personalidad de los mexicanos (Avendaño y Díaz-Guerrero, 1992; Díaz-Guerrero, 1993, 1994; Melgoza y DíazGuerrero, 1990; Palacios y Martínez, 2017), que permitirán comprender de mejor manera su comportamiento en el contexto sociocultural.

Por otra parte, la puntuación promedio de cada faceta se encuentra por arriba de la media teórica, por lo que son interpretadas en el presente estudio como características cardinales en la personalidad de los mexicanos. Considerando la media de cada faceta, la más alta fue la amabilidad, seguida de la complacencia y, por último, la adaptación conductual, lo que apoya lo encontrado por Melgoza y Díaz-Guerrero (1990) respecto al orden de las puntuaciones en cada faceta, además de apoyar la categoría "Amabilidad" de las características de personalidad encontradas por Palacios y Martínez (2017) como la más representativa de la personalidad del mexicano. Estudios correlacionales con instrumentos mexicanos como la abnegación (Avendaño y DíazGuerrero, 1992), los tipos de mexicano (Palacios, 2014), las características de la personalidad del mexicano (Palacios y Martínez, 2017) o el autoconcepto (Díaz-Loving et al., 2002; La Rosa y Díaz-Loving, 1991; Valdez y Reyes, 1993), complementarán la noción sobre rasgos autóctonos (indígenas), posiblemente básicos de la personalidad de los mexicanos propios de esta cultura.

El análisis de consistencia interna para la puntuación total del instrumento mostró coeficientes de confiabilidad cercanos al .85. Las facetas encontradas de la flexibilidad mostraron coeficientes de confiabilidad de entre .66 y .78 . Aunque el índice de confiabilidad para el total del instrumento es aceptable, la fiabilidad para cada faceta se encuentra al límite de lo permitido, atribuido posiblemente a los pocos ítems contenidos en cada faceta. Parece entonces conveniente mejorar el nivel de consistencia interna incorporando nuevas afirmaciones que muestren la variabilidad del constructo encontrado en las facetas de la flexibilidad. 
Es conveniente señalar que el estudio presenta algunas limitaciones a considerar. La primera y posiblemente la más importante corresponde al instrumento, por ejemplo, se deberán revisar los ítems de cada faceta con la finalidad de incorporar una mayor variedad de comportamientos vinculados con rasgos de la cultura mexicana; además de buscar aumentar los niveles de confiabilidad de las tres facetas, particularmente de las facetas de condescendencia y adaptación conductual, ya que se encuentran por debajo de lo aceptable en un instrumento de medida, sobre todo si se pretende vincular la escala obtenida con otros rasgos de personalidad, variables socioculturales o variables conductuales. La segunda es referente al tamaño de la muestra, ya que es relativamente pequeña considerando la población que habita en la zona metropolitana de la Ciudad de México, por lo que para futuros estudios esta se tendrá que ampliar y tener en cuenta que estos resultados no se pueden generalizar a toda la población mexicana, sino que reflejan lo que está pasando con una muestra de jóvenes mexicanos. Considerando el tamaño muestral obtenido, parece conveniente, entonces, que para corroborar los resultados logrados se deberá replicar el estudio en muestras más grandes de diferentes ciudades del país.

El aporte de este estudio fue obtener distintas fuentes de validez de ítems para medir el rasgo de flexibilidad dividido en tres facetas (amabilidad, condescendencia y adaptación conductual), de una manera válida, fiable y culturalmente sensible para medir este tipo de rasgo en una muestra de jóvenes mexicanos. Una segunda contribución fue estimar empíricamente la dirección y magnitud que tiene el rasgo de flexibilidad sobre la eficiencia personal. La última aportación fue proveer evidencia de rasgos centrales de la personalidad de los mexicanos bajo la aproximación etnopsicológica.

Por último, al considerar la forma en que se relacionan las facetas del rasgo de flexibilidad encontradas en esta investigación con la eficiencia personal, se pueden generar las directrices sobre nuevos trabajos en esta línea de investigación. En este sentido, futuros estudios permitirán identificar si la flexibilidad se vincula con la salud física y psicológica del individuo. Además, podremos conocer de qué forma el entorno sociocultural afecta al individuo, ya que colateralmente se tiene una incidencia en la salud, el bienestar y la conducta de las personas.

\section{CONFLICTOS DE INTERÉS}

No existe conflicto de interés por parte del autor.

\section{AGRADECIMIENTOS}

A todos los jóvenes que colaboraron para poder realizar la investigación. 


\section{FINANCIAMIENTO}

El artículo se deriva de la colaboración con la Red de Investigación del Comportamiento del Consumidor, integrada por varias universidades públicas y privadas de México.

\section{REFERENCIAS}

Albanesi, N. S., Garelli, V., y Masramon, M. (2009). Relación entre estilos de personalidad y flexibilidad cognitiva en estudiantes de psicología. Alternativas en Psicología, 14(20), 2-13. http://pepsic.bvsalud.org/scielo.php?pid=S1405339X20090001000 01\&script=sci_abstract\&tlng=en

Arbuckle, J. L., y Wothke, W. (1999). Amos 4.0 users guide. SPSS.

Avendaño, S., y Díaz-Guerrero, R. (1992). Estudio experimental de la abnegación. Revista Mexicana de Psicología, 9(1), 15-19. https://www.redalyc.org/revista.oa?id=2430

Bond, F., Lloyd, J., y Guenole, N. (2013). The Work-Related Acceptance and Action Questionnaire: Initial Psychometric Findings and Their Implications for Measuring Psychological Flexibility in Specific Contexts. Journal of Occupational and Organizational Psychology, 86, 331-347. https://doi.org/10.1111/joop.12001

Buss, D. M. (1991). Evolutionary Personality Psychology. Annual Review of Psychology, 42, 459-491. https://doi.org/10.1146/annurev.ps.42.020191.002331

Buss, D. M. (1996). Social Adaptation and Five Major Factors of Personality. En J. S. Wiggins (Ed.), The Five-Factor Model of Personality: Theorical Perspectives (pp. 180-207). Gilford. https://psycnet.apa.org/record/1996-97942-006

Buss, D. M. (2009). How Can Evolutionary Psychology Successfully Explain Personality and Individual Differences? Perspectives on Psychological Science, 4(4), 359-366. https://doi.org/10.1111/j.1745-6924.2009.01138.x

Buss, D., y Greiling, H. (1999). Adaptative Individual Differences. Journal of Personality, 62(2), 209-243. https://doi.org/10.1111/1467-6494.00053

Cheng, C., y Cheung, M. (2005). Cognitive Processes Underlying Coping Flexibility: Differentiation and Integration. Journal of Personality, 73(4), 859-886. https://doi.org/10.1111/j.1467-6494.2005.00331.x

Church, A. T. (2000). Culture Personality: Toward an Integrated Cultural Trait Psychology. Journal of Personality, 68(4), 651-703. https://doi.org/10.1111/1467-6494.00112

Church, A. T. (2001). Personality Measurement In Cross-Cultural Perspective. Journal of Personality, 69, 979-1006. https://doi.org/10.1111/1467-6494.696172 
Coppens, C., de Boer, S., y Koolhaas, J. (2010). Coping Styles and Behavioural Flexibility: Towards Underlying Mechanisms. Philosophical Transitions, 365, 4021-4028. https://doi.org/10.1098/rstb.2010.0217

Cortés, A. L., y Flores, G. M. (2002). Efectos del sexo, edad y posición ordinal en el autoconcepto en niños. Enseñanza e Investigación en Psicología, 7(1), 117-126. https://go.galegroup.com/ps/anonymous?id=GALE\%7CA116037500\&sid=googl eScholar\&v=2.1\&it=r\&linkaccess=abs\&issn=01851594\&p=IFME\&sw $=w$

Díaz-Guerrero, R. (1967). The Active and the Pasive Syndromes. Revista Interamericana de Psicología, 1(4), 263-272. https://doi.org/10.30849/rip/ijp.v1i4.454

Díaz-Guerrero, R. (1977). A Mexican Psychology. American Psychologist, 32, 934-944. http://dx.doi.org/10.1037/0003-066X.32.11.934

Díaz-Guerrero, R. (1984). La psicología de los mexicanos: un paradigma. Revista Mexicana de Psicología, 1(2), 95-104. https://psycnet.apa.org/record/1987-31268-001

Díaz-Guerrero, R. (1988). Hacia una psicología ecosistémica. Revista de Psicología Social y Personalidad, 4(2), 65-81. http://www.amepso.org/revistas.php?memstatus=2

Díaz-Guerrero, R. (1993). Un factor cardinal en la personalidad de los mexicanos. Revista de Psicología Social y Personalidad, 9(2), 1-19. http://www.amepso.org/revistas. php?memstatus $=2$

Díaz-Guerrero, R. (1994). La psicología del mexicano (6. ${ }^{\text {a }}$ ed.). Trillas.

Díaz-Guerrero, R. (1995). Una aproximación científica a la etnopsicología. Revista Latinoamericana de Psicología, 27(3), 359-389. https://www.redalyc.org/ pdf/805/80527301.pdf

Díaz-Guerrero, R. (1996). La etnopsicología en México. Revista de Psicología Social y Personalidad, 12(1), 1-13. http://www.amepso.org/revistas.php?memstatus=2

Díaz-Guerrero, R. (2001). La teoría del ecosistema humano. En J. Cueli y L. Reidl (Eds.), Teorías de la personalidad (pp. 578-590). Trillas.

Díaz-Guerrero, R. (2008). La psicología del mexicano 2 (2. ${ }^{\text {a }}$ ed.). Trillas.

Díaz-Loving, R. (1998). Contributions of Mexican Ethnopsychology to the Resolution of the Etic-Emic Dilemma in Personality. Journal of Cross-Cultural Psychology, 29(1), 114-118. https://doi.org/10.1177/0022022198291006

Díaz-Loving, R., Reyes-Lagunes, I., y Rivera-Aragón, S. (2002). Autoconcepto: desarrollo y validación de un inventario etnopsicológico. Revista Iberoamericana de Diagnóstico y Evaluación Psicológica, 13, 29-54. https://dialnet.unirioja.es/servlet/ articulo?codigo $=4539939$ 
Flores, G. M., Díaz-Loving, R., y Rivera, A. S. (1987). MERA: Una medida de rasgos asertivos para la cultura mexicana. Revista Mexicana de Psicología, 4(1), 29-40. https://www.redalyc.org/revista.oa?id=2430

Hair, J., Anderson, R., Tatham, R., y Black, W. (1999). Análisis multivariante (5. a ed.). Prentice Hall.

La Rosa, J., y Díaz-Loving, R. (1991). Evaluación del autoconcepto: una escala multidimensional. Revista Latinoamericana de Psicología, 23, 15-33. https://www. redalyc.org/pdf/805/80523102.pdf

La Rosa, J., Díaz-Loving, R., y Andrade, P. P. (1986). Escalas de locus de control: problemas y contribuciones. Revista Mexicana de Psicología, 3(2), 150-153. https://www. redalyc.org/revista.oa?id=2430

Martínez, A. R., Hernández, LI. M., y Hernández, LI. V. (2006). Psicometría. Alianza.

McCrae, R. (2001). Trait Psychology and Culture: Exploring Intercultural Comparisons. Journal of Personality, 69(6), 819-846. https://doi.org/10.1111/1467-6494.696166

Melgoza, E. E., y Díaz-Guerrero, R. (1990). El desarrollo de una escala de flexibilidad en sujetos mexicanos. La Psicología Social en México, 3, 20-24. http://www.amepso. org/publicaciones.php?bkt_id=2

Montero, I., y León, 0. (2002). Clasificación y descripción de las metodologías de investigación en psicología. Revista Internacional de Psicología Clínica y de la Salud, 2(3), 503-508. https://www.redalyc.org/pdf/337/33720308.pdf

Ortiz, F., Church, T., Vargas, J., Ibáñez, J., Flores, M., luit, J., y Escamilla, J. (2007). Are Indigenous Personality Dimensions Culture-Specific? Mexican Inventories and the Five-Factor Model. Journal of Research in Personality, 41, 618-649. https://doi. org/10.1016/j.jrp.2006.07.002

Palacios, D. J. (Septiembre del 2014). Evaluación de los rasgos de personalidad del mexicano. Segundo Coloquio de Psicología efectuado en la Universidad Humanitas, México.

Palacios, D. J. (2015). Propiedades psicométricas del inventario de búsqueda de sensaciones para adolescentes en México (IBS-Mx). International Journal of Psychological Research, 8(1), 46-60. https://doi.org/10.21500/20112084.644

Palacios, D. J., Bustos, A. J. M., y Mercado D. S. (2013). Implicaciones de las estrategias de adaptación sobre la conducta proambiental en jóvenes. Revista Psicología Iberoamericana, 21(1), 65-78. https://www.redalyc.org/pdf/1339/133929862008. pdf 
Palacios, D. J., Bustos, J. M., y Soler, A. L. (2015). Factores socioculturales vinculados al comportamiento proambiental en jóvenes. Revista de Psicología, 24(1), 1-16. https://doi.org/10.5354/0719-0581.2015.36900

Palacios, D. J., y Martínez, M. R. (2017). Descripción de características de personalidad y dimensiones socioculturales en jóvenes mexicanos. Revista de Psicología, 35(2), 453-484. http://dx.doi.org/10.18800/psico.201702.003

Palacios, D. J., Ramírez, A. V., Hernandez, M. H., y Anaya, L. A. (2016). Neuroasociaciones del consumo de bebidas endulzadas. Revista Mexicana de Neurociencias, 17(6), 64-71. http://previous.revmexneurociencia.com/wp-content/uploads/2016/11/ RevMexNeu-No-6-Nov-Dec-2016-64-71-CO.pdf

Palacios, J., y Soler, A. L. (2017). Preliminary Study of the Comparative Profile of Financial Control in University Young People. Ciencias Psicológicas, 11(2), 201-212. https:// doi.org/10.22235/cp.v11i2

Pérez, G. J., Chacón, M. S., y Moreno, R. R. (2000). Validez de constructo: el uso de análisis factorial exploratorio-confirmatorio para obtener evidencias de validez. Psicothema, 12(2), 442-446. http://www.psicothema.com/psicothema. asp?id=601

Real Academia de la Lengua Española (2015). Definición de flexibilidad. http://lema.rae. es/drae/?val=flexibilidad

Secretaría de Salud (2011). Reglamento de la Ley General de Salud en Materia de Investigación para la Salud. http://www.salud.gob.mx/unidades/cdi/nom/compi/rlgsmis.html

Reyes, L. I. (1996). La medición de la personalidad en México. Revista de Psicología Social y Personalidad, 12, 31-59.

Roberts, M., Barthel, F., López, C., Tchanturia, K., y Treasure, J. (2011). Development and Validation of the Detail and Flexibility Questionnaire (DFlex) in Eating Disorders. Eating Behaviors, 12, 168-174. https://doi.org/10.1016/j.eatbeh.2011.04.001

Singelis, T. M., Triandis, H. C., Bhawuk, D. P. S., y Gelfand, M. J. (1995). Horizontal and Vertical Dimensions of Individualism and Collectivism: A Theoretical and Measurement Refinement. Cross-Cultural Research, 29(3), 240-275. https://doi. org/10.1177/106939719502900302

Triandis, H. C. (1994). Culture and Social Behavior. McGraw-Hill.

Triandis, H. C. (1996). The Psychological Measurement of Cultural Syndromes. American Psychologist, 51(4), 407-415. https://psycnet.apa.org/buy/1996-04055-028

Triandis, H. C. (2000). Culture and Conflict. International Journal of Psychology, 35(2), 145-152. https://doi.org/10.1080/002075900399448 
Triandis, H. C. (2001). Individualism-Collectivism and Personality. Journal of Personality, 69(6), 907-924. https://doi.org/10.1111/1467-6494.696169

Valdez, M. J., González, A. N., Jiménez, H. M., y Cañas, M. J. (1996). El autoconcepto en Chiapanecos. La Psicología Social en México, 6, 64-70. http://www.amepso.org/ publicaciones.php?bkt_id=2

Valdez, M. J., y Reyes, L. I. (1992). Las categorías semánticas y el autoconcepto. La Psicología Social en México, 4, 193-199. http://www.amepso.org/publicaciones. php?bkt_id=2

Valdez, M. J., y Reyes, L. I. (1993). La construcción de instrumentos de medición a partir de categorías semánticas. Un caso ilustrativo: el autoconcepto. Revista de Psicología Social y Personalidad, 9(1), 57-66. http://www.amepso.org/publicaciones. php?bkt_id=2

Varela-Macedo, V. M., Díaz-Loving, R., y Reyes-Lagunes, I. (1998). Aculturación de estudiantes extranjeros a México: aplicación del escalamiento multidimensional. La Psicología Social en México, 7, 24-29. http://www.amepso.org/publicaciones. php?bkt_id=2

Williams, K., Ciarrochi, J., y Heaven, P. (2012). Inflexible Parents, Inflexible Kids: A 6-year Longitudinal Study of Parenting Style and the Development of Psychological Flexibility in Adolescents. Journal of Youth Adolescence, 41(8), 1053-66. https:// link.springer.com/article/10.1007/s10964-012-9744-0 